\title{
Motives to Report Adverse Drug Reactions to the National Agency: A Survey Study among Healthcare Professionals and Patients in Croatia, The Netherlands, and the UK
}

\author{
Sieta T. de Vries ${ }^{1}$ (D) - Petra Denig ${ }^{1}$ (1) - Adriana Andrić ${ }^{2}$ (I) - Marina Dimov Di Giusti ${ }^{2}$ - Alicia Ptaszynska-Neophytou ${ }^{3}$. \\ Linda Härmark ${ }^{4}$. Peter G. M. Mol ${ }^{1,5}$ iD on behalf of the IMI Web-RADR Work Package 3b Consortium and SCOPE \\ Joint Action Work Package 4
}

Accepted: 4 July 2021 / Published online: 8 August 2021

(c) The Author(s) 2021

\begin{abstract}
Introduction Healthcare professionals (HCPs) and patients have various motives to report adverse drug reactions (ADRs) to their national agency. These motives may differ between countries.

Objective The aim of this study was to assess to what extent motives of HCPs and patients to report ADRs differ between countries.

Methods HCPs and patients from Croatia (HR), The Netherlands (NL), and the UK were asked to complete a web-based survey containing questions regarding demographics and ADR reporting. HCPs and patients could select all motives for reporting that applied to them, with a total of 23 and 24 motives, respectively. Descriptive statistics are presented and Chisquare tests were used to test for differences across the countries, with effect sizes calculated using Cramer's $V$.

Results In total, 296 HCPs and 423 patients were included (60\% and 32\% from Croatia, 19\% and 44\% from NL, and $21 \%$ and $24 \%$ from the UK, respectively). For most of the motives to report or not to report an ADR, there were no differences between countries. Most HCPs from all countries would be motivated to report an ADR if there was a strong suspicion of causality (89\%), if it concerned a severe/serious ADR (86\%), and if it concerned an ADR for a new, recently marketed drug (77\%). Most patients from all countries agreed that they would report an ADR if it concerned a severe ADR (96\%), if the ADR influenced their daily activities (91\%), and if they were worried about their own situation (90\%). Differences across the countries $(p<0.05$ and $V \geq 0.21)$ were observed for three and four of the HCP and patient motives, respectively. For HCPs, these differences were seen in motives related to legal obligation $(65 \% \mathrm{HR}, 24 \% \mathrm{NL}, 38 \%$ UK), black triangle medicines ( $27 \% \mathrm{HR}, 4 \% \mathrm{NL}, 77 \% \mathrm{UK}$ ), and the reporting of well-known ADRs (53\% HR, 85\% NL, 69\% UK). For patients, these differences were seen in motives related to a linkage between the ADR report and the medical notes $(59 \% \mathrm{HR}, 60 \%$ NL, 30\% UK), complexity and time taken to report ( $25 \% \mathrm{HR}, 13 \% \mathrm{NL}, 40 \% \mathrm{UK})$, medicines purchased on the internet $(59 \% \mathrm{HR}, 39 \%$ NL, 65\% UK), and the reporting of embarrassing ADRs (32\% HR, $11 \% \mathrm{NL}, 35 \% \mathrm{UK})$.

Conclusions HCPs' and patients' motives to report or not to report ADRs to the national agency were mostly similar across the three countries. Such motives can be used in general strategies to promote and increase ADR reporting. The observed differences provide guidance to further fine-tune ADR reporting at a national level.
\end{abstract}

\section{Introduction}

Postmarketing surveillance is crucial for monitoring a drug's safety profile after its market approval. Worldwide, spontaneous reporting is the leading method for this surveillance

Peter G. M. Mol

p.g.m.mol@umcg.nl

Extended author information available on the last page of the article
[1]. Currently, many national drug regulatory agencies across the world allow both patients and healthcare professionals (HCPs) to report adverse drug reactions (ADRs) directly to the agency [2]. One of the limitations of spontaneous reporting is underreporting of ADRs. A systematic review showed high levels of underreporting, ranging from 82 to $98 \%$ across the included studies [3]. ADR reporting rates are vastly different across countries. A study evaluating all ADR reports to the Uppsala Monitoring Centre (UMC) between 2000 and 2009 showed that six countries 


\section{Key Points}

Differences between Croatia, The Netherlands, and the UK in motives to report adverse drug reactions (ADRs) to the national agency were observed in 3 of the 23 motives and 4 of the 24 motives assessed in a survey study among healthcare professionals (HCPs) and patients, respectively.

For HCPs, differences across the countries in motives to report related to legal obligation, black triangle medicines, and the reporting of well-known ADRs.

For patients, differences across the countries in motives to report related to linkage between the report and the medical notes, complexity and time taken to report, medicines purchased on the internet, and the reporting of embarrassing ADRs.

Besides general ADR awareness and reporting strategies, country-specific strategies could be used to promote and increase the reporting of ADRs by HCPs and patients.

contributed $65 \%$ of all reports from 96 countries [4]. The UK and The Netherlands are countries with a high number of reports from patients $[5,6]$.

Various studies have assessed HCPs' motives to report or not to report ADRs [7-16], including some systematic reviews [17-20]. Although direct patient reporting has been introduced more recently [21-23], studies have also assessed patients' motives to report or not to report ADRs [24-27]. Some of the identified motives among HCPs and patients are related to the reporter, such as a lack of knowledge, while other motives are related to the specific case, such as whether it concerns a serious and/or severe ADR or a previously unknown ADR. Finally, some motives are related to the system or the process of reporting, such as accessibility of the ADR forms and time taken to report.

To date, little attention has been paid to potential differences in motives to report or not to report ADRs across countries. Such information is relevant for adapting national ADR reporting systems and developing support tools for ADR reporting. The Web-Recognizing Adverse Drug Reactions (Web-RADR) mobile application (app) for ADR reporting was introduced in three European countriesCroatia, The Netherlands, and the UK [28]. The app could potentially solve some of the reasons not to report an ADR, such as no easy access to ADR forms or a lack of time [29]. It was found that HCPs' and patients' perceptions towards the app and their willingness to use it differed across the countries [30], which suggests that different strategies across countries are needed to promote and increase ADR reporting. The aim of this study was to contribute to strategies for improving ADR reporting by assessing to what extent motives of HCPs and patients to report or not to report ADRs differ between Croatia, The Netherlands, and the UK.

\section{Methods}

This study was a collaboration between the Innovative Medicines Initiative (IMI) Web-RADR project (https://web-radr. eu/) [29] and the Strengthening Collaboration for Operating Pharmacovigilance in Europe (SCOPE) Joint Action [31]. In the context of the Web-RADR project, a web-based survey was developed in the Croatian, Dutch, English, French, German, Portuguese, and Spanish languages to collect information from European HCPs and patients about their preferences towards a mobile app on two-way risk communication about medicines. Additional questions about reporting of ADRs to the national agency were included in the survey by members of the SCOPE Joint Action. HCPs and patients from European countries could participate in the study, but the survey distribution particularly focused on reaching responders from Croatia, The Netherlands, and the UK. More detailed information about the survey, its development, and its distribution is presented elsewhere [30].

Responders who completed the Croatian, Dutch, or English version of the survey and were living in Croatia, The Netherlands, or the UK, respectively, were included in this study. The following HCPs were included: primary care physicians, community/primary care pharmacists, hospital pharmacists, nurse practitioners, secondary care/hospital physicians/medical specialists, and support staff (e.g. prescribing advisor, pharmacy dispenser, pharmacy technician, hospital nurse, pharmaceutical manager).

\subsection{Survey Questions}

\subsubsection{Demographic Questions}

For HCPs, the following demographic information was collected to describe the responders: age, sex, years of accreditation, and whether they had ever reported an ADR to the national agency. For patients, the following information was collected: age, sex, educational level, number of prescribed medicines, whether they had ever experienced an ADR, and whether they were aware of the possibility to report ADRs to the national agency.

\subsubsection{Questions About Motives to Report or Not to Report Adverse Drug Reactions (ADRs)}

One question asked HCPs about their motives to report ADRs to the national agency, i.e. 'What would motivate 
you to report an ADR?', while another question asked about motives not to report an ADR, i.e. 'What are your reasons for not reporting an ADR?'. For both questions, answer options were based on previous studies [3, 32]. There were 23 answer options assessing HCPs' motives to report; 14 motives to report and 9 motives not to report. HCPs could select any of the motives that applied to them and provide an additional open-ended answer, or select 'nothing would motivate me'/'none'.

The patient survey contained one question asking about the circumstances when they would report an ADR to the national agency. For this question, 13 statements were presented starting with 'I would report a side effect to the national agency if ...'. Patients were asked to answer each statement on a five-point Likert scale ranging from totally agree to totally disagree. A second question asked about the patient's perspectives on the benefits of reporting ADRs directly to the national agency, i.e. 'In your opinion, what are the benefits of reporting side effects directly to the national agency?'. A third question was included about the drawbacks of ADR reporting, i.e. 'What are, in your opinion, the drawbacks of reporting side effects directly to the national agency?'. Patients could select multiple benefits and drawbacks and provide an additional open-ended benefit or drawback, or select 'none'. The statements and answer options used in these questions were based on a previous study [25]. There were 24 statements and answer options assessing patients' motives; 18 motives to report (13 statements and 5 benefits) and 6 motives not to report (drawbacks of reporting).

\subsection{Analyses}

Descriptive results are presented per country for both HCPs and patients. The results of the statements in the patient survey are presented as a percentage of responders that agreed or totally agreed with the statement. Answers given by responders to open-ended fields were classified as one of the existing answer options, where possible, by one researcher (STdV) and checked by another (PGMM), and otherwise considered as 'other'.

Next, differences in each of the HCP and patient motives to report or not to report ADRs across the countries were tested using Chi-square tests. $p$ values $<0.05$ were considered statistically significant. For motives that were statistically significant, post hoc pairwise comparisons using Chi-square tests were conducted to assess which countries differed significantly from each other. The Bonferroni method was used in these analyses to adjust for multiple testing. This implies that $p$ values $<0.017$ ( $p$ value $0.05 / 3$-country comparisons) were considered statistically significant. In addition, the size of the effect was calculated for statistically significant motives. For this, Cramer's $V$ was used, where two degrees of freedom values of $\geq 0.21$ and $\geq 0.35$ were considered medium and large, respectively [33].

The data were analyzed using Stata version 13 (StataCorp LLC, College Station, TX, USA) and Microsoft Excel 2010 (Microsoft Corporation, Redmond, WA, USA) was used for a graphical presentation of the results.

\section{Results}

\subsection{Healthcare Professionals (HCPs)}

In total, 296 HCPs were included. Their median age was 37 years and two-thirds were female $(68 \%)$ and one-third were pharmacists (34\%). Most HCPs had reported an ADR to the national agency $(81 \%)$ [Electronic Supplementary Material (ESM) Table 1]. Of the HCPs, $60 \%$ were from Croatia, $19 \%$ were from The Netherlands, and $21 \%$ were from the UK. HCPs from Croatia had reported an ADR to the pharmacovigilance center more often than HCPs from the other countries. HCPs from The Netherlands were somewhat younger and had fewer years of accreditation than HCPs from Croatia and the UK. HCPs from the UK were more often males than HCPs from the other countries (Table 1).

\subsubsection{HCPs' Motives to Report or Not to Report an ADR}

Most HCPs from all countries would be motivated to report an ADR to the national agency if there was a strong suspicion of causality (overall $89 \%$, range from $85 \%$ in The Netherlands to $91 \%$ in Croatia), if it concerned a severe and/ or serious ADR (overall $86 \%$, range from $84 \%$ in Croatia to $90 \%$ in the UK), and if it concerned an ADR for a new, recently marketed drug (overall $77 \%$, range from $72 \%$ in Croatia to $90 \%$ in the UK) [ESM Table 2a].

In all countries, HCPs' motives not to report an ADR to the national agency were mostly a lack of time (overall $64 \%$, range from $54 \%$ in the UK to $68 \%$ in Croatia) and if it concerned a well-known ADR (overall 63\%, range from 53\% in Croatia to $85 \%$ in The Netherlands) [ESM Table 2b].

\subsubsection{HCPs-Differences Across the Countries}

Overall, statistically significant differences across the countries were shown in 10 of the 23 assessed motives among the HCPs. Eight of the differences were shown in the motives to report (Fig. 1a) and two were shown in the motives not to report (Fig. 1b). Three of the 10 motives with statistically significant differences across the countries had a medium or large effect size.

Of the motives to report, differences with a large or medium effect size were seen for the reporting of an ADR for a medicine with a black triangle $(V=0.52)$ and if there 
Table 1 Characteristics of the included healthcare professionals, by country

\begin{tabular}{llll}
\hline & Croatia & The Netherlands & UK \\
\hline$n(\%)$ & $177(60)$ & $57(19)$ & $62(21)$ \\
Median age, years (range) $^{\mathrm{a}}$ & $38(20-71)$ & $35(21-60)$ & $37(20-65)$ \\
Sex $^{\mathrm{b}}$ & & & \\
Male & $46(26)$ & $19(33)$ & $28(45)$ \\
Female & $130(74)$ & $37(65)$ & $34(55)$ \\
Other/prefer not to say & $0(0)$ & $1(2)$ & $0(0)$ \\
Profession & & & $1(2)$ \\
Primary care physician & $28(16)$ & $6(11)$ & $22(35)$ \\
Community/primary care pharmacist & $57(32)$ & $22(39)$ & $18(29)$ \\
Hospital pharmacist & $15(8)$ & $9(16)$ & $7(11)$ \\
Nurse practitioner & $13(7)$ & $4(7)$ & $9(15)$ \\
Secondary care/hospital physician/medical specialist & $64(36)$ & $6(11)$ & $5(8)$ \\
Support staff & $0(0)$ & $10(18)$ & $11(18)$ \\
Accreditation, years & & & $32(52)$ \\
$<5$ & $43(24)$ & $24(42)$ & $19(31)$ \\
$5-20$ & $86(49)$ & $19(33)$ & $44(71)$ \\
$>20$ & $48(27)$ & $14(25)$ & $16(26)$ \\
Ever reported an ADR to the national pharmacovigilance center & $151(85)$ & $44(77)$ & $11(19)$ \\
Yes & $17(10)$ & $2(4)$ & \\
No & $9(5)$ & & \\
Do not know/do not remember & & \\
\hline
\end{tabular}

Data are expressed as $n(\%)$ unless otherwise specified

$A D R$ adverse drug reaction

${ }^{\text {a }}$ wwo missing from Croatia

${ }^{\mathrm{b}}$ One missing from Croatia was a legal obligation to report $(V=0.34)$. More HCPs from the UK would be motivated to report an ADR for a medicine with a black triangle than HCPs from Croatia and The Netherlands (77\%, 27\%, and 4\%, respectively; $p<0.001$ for each specific comparison between two countries). A legal obligation would motivate more HCPs from Croatia than from the UK and The Netherlands $(65 \%, 38 \%$, and $24 \%$, respectively; $p<0.001$ for the comparison of Croatia with The Netherlands, and Croatia with the UK) (Fig. 1a; ESM Table 2a).

The motive not to report, with a medium effect size, was if it concerned a well-known ADR $(V=0.26)$. HCPs from The Netherlands selected this motive more often than HCPs from Croatia (85\% vs. 53\%; $p<0.001$ ) (Fig. 1b; ESM Table 2b).

\subsection{Patients}

In total, 423 patients were included, with a median age of 51 years. Almost two-thirds were female (62\%), most had experienced an ADR (81\%), and more than half were aware of the possibility to report ADRs to the national pharmacovigilance center (54\%) (ESM Table 3). Of the patients,
$32 \%$ were from Croatia, $44 \%$ were from The Netherlands, and $24 \%$ were from the UK. Patients from The Netherlands were older, were more often males, and were more often taking five or more prescribed medicines than the patients from Croatia and the UK. The number of patients without any prescribed medicine was largest among responders from Croatia, while the largest number of patients aware of the possibility to report ADRs to the national pharmacovigilance center was shown among responders from the UK (Table 2).

\subsubsection{Patients' Motives to Report or Not to Report an ADR}

Most patients from all countries agreed that they would report an ADR to the national agency if it concerned a severe ADR (overall $96 \%$, range from $92 \%$ in the UK to $99 \%$ in Croatia), if the ADR influenced their daily activities (overall $91 \%$, range from $88 \%$ in The Netherlands to $96 \%$ in Croatia), if they were worried about their own situation (overall 90\%, range from $86 \%$ in the UK to $96 \%$ in Croatia), and if the ADR was not mentioned in the patient information leaflet (overall $87 \%$, range from $85 \%$ in the UK to $90 \%$ in Croatia) (ESM Table 4 and Table 3). The benefits for patients from 


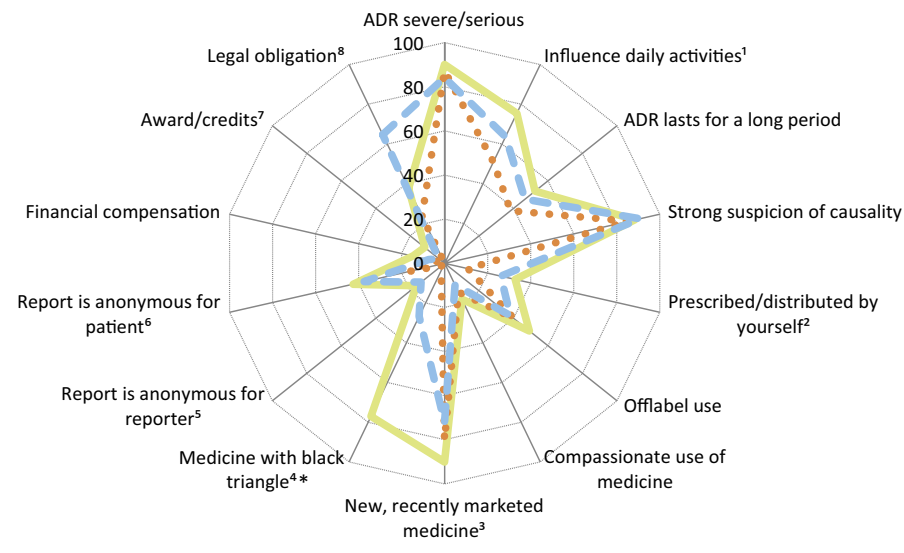

(A)

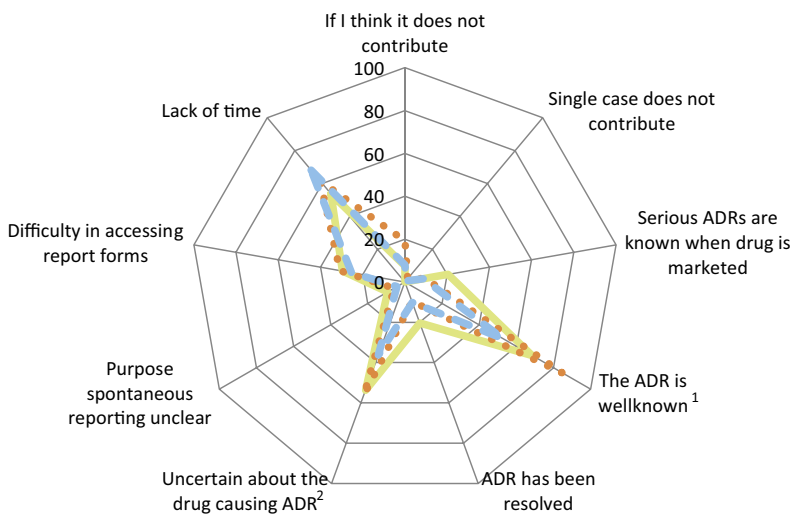

(B)
Fig. 1 Healthcare professionals' responses to the question a 'What would motivate you to report an adverse drug reaction?' (3 missing; 1 'nothing would motivate me'; 11 other answers), and $\mathbf{b}$ 'What are your reasons for not reporting an ADR?' (48 'none'; 9 other answers). *A black triangle is assigned to medicinal products that are subjected to additional safety monitoring [34]. ADR adverse drug reaction. Fig. 1A: ${ }^{1}$ Overall $\mathrm{P}=0.023$. $\mathrm{V}=0.16$. The Netherlands and the UK significantly different $(\mathrm{P}=0.006) .{ }^{2}$ Overall $\mathrm{P}=0.019$. $\mathrm{V}=0.16$. The Netherlands significantly different the UK $(\mathrm{P}=0.005)$. ${ }^{3}$ Overall $\mathrm{P}=0.013$. $\mathrm{V}=0.17$. Croatia and the UK significantly different $(\mathrm{P}=0.004) .{ }^{4}$ Overall $\mathrm{P}<0.001 . \mathrm{V}=0.52 . \mathrm{P}<0.001$ for each

all countries to report ADRs were mostly that they could contribute to drug safety (overall $85 \%$, range from $80 \%$ in The Netherlands to $95 \%$ in the UK) and share their experiences for the benefit of others (overall $79 \%$, range from $76 \%$ in Croatia and The Netherlands to $90 \%$ in the UK) (ESM Table 5a).

Motives for not reporting an ADR to the national agency selected by many patients from all countries were not knowing what happens with the report (overall 63\%, range from $59 \%$ in The Netherlands to $70 \%$ in Croatia) and not knowing how to report (overall $47 \%$, range from $43 \%$ in The Netherlands to $52 \%$ in the UK (ESM Table 5b).

\subsubsection{Patients-Differences Across the Countries}

Overall, statistically significant differences for patients across countries were shown in 11 of the 24 assessed motives (Fig. 2, Table 3). Ten of the differences were shown in the motives to report and one in the motives not to report. Four of the 11 motives with statistically significant differences across the countries had a medium effect size.

Of the motives to report, differences with a medium effect size were seen for the following motives; if the report would not be linked to the medical notes to which HCPs have access $(V=0.25)$, if the medicine is purchased on the internet instead of in the pharmacy $(V=0.23)$, and the benefit of specific comparison between two countries. ${ }^{5}$ Overall $\mathrm{P}=0.031$. $\mathrm{V}=0.15$. The Netherlands significantly different from Croatia $(\mathrm{P}=0.014)$ and the UK $(\mathrm{P}=0.007) .{ }^{6}$ Overall $\mathrm{P}=0.005 . \mathrm{V}=0.19$. The Netherlands significantly different from Croatia $(\mathrm{P}=0.003)$ and the UK $(\mathrm{P}=0.002) .{ }^{7}$ Overall $\mathrm{P}=0.006 . \mathrm{V}=0.18$. The UK and the Netherlands significantly different $(\mathrm{P}=0.010) .{ }^{8}$ Overall $\mathrm{P}<0.001$. $\mathrm{V}=0.34$. Croatia significantly different from the Netherlands and the UK $\left(\mathrm{P}<0.001\right.$ for both). Fig. 1B: ${ }^{1}$ Overall $\mathrm{P}<0.001$. $\mathrm{V}=0.26$. Croatia and the Netherlands significantly different $(\mathrm{P}<0.001) .{ }^{2}$ Overall $\mathrm{P}=0.015 . \mathrm{V}=0.18$. Croatia and the Netherlands significantly different $(\mathrm{P}=0.015)$

reporting ADRs that patients are too embarrassed to discuss with HCPs $(V=0.27)$. More patients from Croatia and The Netherlands found it important that the report was not linked to their medical notes, when compared with the UK (59\%, $60 \%$, and $30 \%$, respectively; $p<0.001$ for the comparison of Croatia and the UK, and The Netherlands and the UK). Fewer patients from The Netherlands than from Croatia and the UK indicated they would report if they had purchased the medicine on the internet instead of from a pharmacy (39\%, $59 \%$, and $65 \%$, respectively; $p<0.001$ for the comparison between The Netherlands and Croatia, and The Netherlands and the UK) (Table 3). Furthermore, fewer patients from The Netherlands than from Croatia and the UK saw it as a benefit to be able to report embarrassing ADRs directly to a national agency $(11 \%, 32 \%$, and $35 \%$, respectively; $p<0.001$ for the comparison between The Netherlands and Croatia, and between The Netherlands and the UK) (Fig. 2a, ESM Table 5a).

Of the motives not to report, the motive that reporting is too complicated/time-consuming showed a difference across countries with a medium effect size $(V=0.25)$. This was considered to be less of an issue for patients from The Netherlands than patients from Croatia and the UK (13\% of the patients in The Netherlands versus $25 \%$ of the patients in Croatia $[p=0.013]$ and $40 \%$ of the patients in the UK $[p<0.001]$ ) (Fig. 2b, ESM Table 5b). 
Table 2 Characteristics of the included patients, by country

\begin{tabular}{|c|c|c|c|}
\hline & Croatia & The Netherlands & UK \\
\hline$n(\%)$ & $136(32)$ & $187(44)$ & $100(24)$ \\
\hline Median age, years (range) ${ }^{\mathrm{a}}$ & $37(14-68)$ & $60(20-89)$ & $48(21-74)$ \\
\hline \multicolumn{4}{|l|}{ Sex } \\
\hline Male & $39(29)$ & $91(49)$ & $29(29)$ \\
\hline Female & $97(71)$ & $96(51)$ & $69(69)$ \\
\hline Other/prefer not to say & $0(0)$ & $0(0)$ & $2(2)$ \\
\hline \multicolumn{4}{|l|}{ Education } \\
\hline Primary education & $3(2)$ & $0(0)$ & $0(0)$ \\
\hline Lower secondary education & $10(7)$ & $25(13)$ & $4(4)$ \\
\hline Upper secondary education & $30(22)$ & $29(16)$ & $10(10)$ \\
\hline Post-secondary but non-tertiary education & $11(8)$ & $27(14)$ & $5(5)$ \\
\hline First stage of tertiary education & $26(19)$ & $73(39)$ & $42(42)$ \\
\hline Second stage of tertiary education & $56(41)$ & $33(18)$ & $39(39)$ \\
\hline \multicolumn{4}{|l|}{ Number of prescribed medicines } \\
\hline 0 & $59(43)$ & $6(3)$ & $15(15)$ \\
\hline 1 & $23(17)$ & $16(9)$ & $15(15)$ \\
\hline 2 & $20(15)$ & $20(11)$ & $12(12)$ \\
\hline 3 & $9(7)$ & $9(5)$ & $15(15)$ \\
\hline 4 & $8(6)$ & $27(14)$ & $8(8)$ \\
\hline 5 or more & $17(13)$ & $109(58)$ & $35(35)$ \\
\hline \multicolumn{4}{|l|}{ Experienced an adverse effect } \\
\hline Yes & $102(75)$ & $159(85)$ & $80(80)$ \\
\hline No & $24(18)$ & $23(12)$ & $14(14)$ \\
\hline Do not know/do not remember & $10(7)$ & $5(3)$ & $6(6)$ \\
\hline \multicolumn{4}{|c|}{ Aware of the possibility to report ADRs to the national pharmacovigilance centre } \\
\hline Yes & $87(64)$ & $72(39)$ & $69(69)$ \\
\hline No & $33(24)$ & $55(29)$ & $19(19)$ \\
\hline I have never heard of the national pharmacovigilance centre & $16(12)$ & $60(32)$ & $12(12)$ \\
\hline
\end{tabular}

Data are expressed as $n(\%)$ unless otherwise specified

$A D R$ adverse drug reaction

${ }^{\mathrm{a} O n e}$ missing from The Netherlands

\section{Discussion}

Differences across the countries in the proportion of HCPs and patients agreeing with the motives to report or not to report ADRs to the national agency were, in general, small. No differences were seen for the main motives for HCPs to report an ADR, i.e. if there was a strong suspicion of causality and if it concerned a severe and/or serious ADR. Furthermore, for patients, no diferences across countries were seen in their main motives to report, such as whether it concerned ADRs influencing their daily activities or worrisome ADRs, or in common reasons not to report, including not knowing what happens with the report and not knowing how to report. However, differences were seen in 3 of all 23 motives and 4 of all 24 motives assessed among HCPs and patients, respectively. For HCPs, differences were related to legal obligation, black triangle medicines, and the reporting of well-known ADRs, and for patients, differences were related to a linkage between the report and the medical notes, medicines purchased on the internet, the reporting of embarrassing ADRs, and the complexity and time taken to report.

This study illustrates that some of the motives to report or not to report ADRs, as mentioned in previous studies, may apply, to a different extent, to HCPs or patients from different countries. This finding is in line with a previous study regarding HCPs in nine European countries showing that there are differences across countries in factors for the decision to report an ADR [35]. An example from the previous study is that the importance of confidence in the diagnosis of the ADR ranged from $29 \%$ of the responders from Portugal to $76 \%$ of the responders from Spain [35], and was 34\% and $49 \%$ of the responders from The Netherlands and the UK, 
Table 3 Number (\%) of patients who agreed or totally agreed per statement, starting with 'I would report a side effect to the national agency ...', by country and $p$ values for tests of differences across the countries

\begin{tabular}{|c|c|c|c|c|}
\hline & Croatia & The Netherlands & UK & $p$ Value \\
\hline$\ldots$ if the side effect was severe & $135(99)$ & $177(95)$ & $92(92)$ & $\begin{array}{l}0.022(V=0.13) \\
\text { HR-NL: } 0.024 \\
\text { NL-UK: } 0.377 \\
\text { HR-UK: } \mathbf{0 . 0 0 4}\end{array}$ \\
\hline$\ldots$ if the side effect influences my daily activities & $130(96)$ & $165(88)$ & $90(90)$ & 0.068 \\
\hline$\ldots$ if the side effect is not mentioned in the PIL & $122(90)$ & $163(87)$ & $85(85)$ & 0.551 \\
\hline$\ldots$ if I am worried about my own situation & $130(96)$ & $165(88)$ & 86 (86) & $\begin{array}{l}0.028(V=0.13) \\
\text { HR-NL: } 0.020 \\
\text { NL-UK: } 0.586 \\
\text { HR-UK: } \mathbf{0 . 0 0 9}\end{array}$ \\
\hline$\ldots$ if the side effect has not yet been resolved & $127(93)$ & $154(82)$ & 84 (84) & $\begin{array}{l}0.013(V=0.14) \\
\text { HR-NL: } \mathbf{0 . 0 0 4} \\
\text { NL-UK: } 0.724 \\
\text { HR-UK: } 0.021\end{array}$ \\
\hline$\ldots$ if I am completely sure that the symptom is a side effect of my medicine & $122(90)$ & $157(84)$ & $84(84)$ & 0.288 \\
\hline $\begin{array}{l}\ldots \text { if the side effect lasts for a relatively long period (for instance more than two/three } \\
\text { days) }\end{array}$ & $122(90)$ & $148(79)$ & $84(84)$ & $\begin{array}{l}0.040(V=0.14) \\
\text { HR-NL: 0.011 } \\
\text { NL-UK: } 0.319 \\
\text { HR-UK: } 0.193\end{array}$ \\
\hline$\ldots$ if the side effect is caused by a medicine prescribed by an HCP & $120(88)$ & $155(83)$ & $85(85)$ & 0.411 \\
\hline$\ldots$ if I asked an HCP but he/she refused & $115(85)$ & $160(86)$ & $86(86)$ & 0.947 \\
\hline $\begin{array}{l}\ldots \text { if the side effect is caused by a medicine that I use without a prescription from an } \\
\text { HCP }\end{array}$ & $94(69)$ & $88(47)$ & $61(61)$ & $\begin{array}{l}<0.001(V=0.20) \\
\text { HR-NL: }<\mathbf{0 . 0 0 1} \\
\text { NL-UK: } 0.024 \\
\text { HR-UK: } 0.194\end{array}$ \\
\hline$\ldots$ if I have purchased the medicine on the internet, not in the pharmacy & $80(59)$ & $72(39)$ & $65(65)$ & $\begin{array}{l}<0.001(V=0.23) \\
\text { HR-NL: }<\mathbf{0 . 0 0 1} \\
\text { NL-UK: }<\mathbf{0 . 0 0 1} \\
\text { HR-UK: } 0.335\end{array}$ \\
\hline$\ldots$ if completing the report takes only a few minutes of my time & $105(77)$ & $128(68)$ & $79(79)$ & 0.083 \\
\hline$\ldots$ if the report will not be linked to my medical notes to which HCPs have access & $80(59)$ & $113(60)$ & 30 (30) & $\begin{array}{l}<0.001(V=0.25) \\
\text { HR-NL: } 0.772 \\
\text { NL-UK: }<\mathbf{0 . 0 0 1} \\
\text { HR-UK: }<\mathbf{0 . 0 0 1}\end{array}$ \\
\hline
\end{tabular}

$H C P$ healthcare professional, $P I L$ patient information leaflet, $H R$ Croatia, $N L$ The Netherlands

respectively. Our study showed that the percentage of HCPs being motivated to report an ADR when there was a strong suspicion of causality ranged from $85 \%$ in The Netherlands to $91 \%$ in Croatia, and was $89 \%$ in the UK. The previous study was conducted in 1994 . The comparison with the current study suggests that the differences across the countries may have become smaller and that the motivation of HCPs to report ADRs may have changed over time.

Motives with differences across the countries in our study for HCPs and patients were mostly related to the specific case and the healthcare system. This might be explained in part by differences in the ADR reporting system across the countries. For instance, there are differences between Croatia, The Netherlands, and the UK in the number of fields to be completed in the ADR reporting form $(24,41$, and 53 fields, respectively) [6]. Additionally, other aspects, such as differences in promotional activities towards ADR reporting, system maturity, and differing health care settings across the three countries, have been mentioned [36]. Furthermore, there are differences in reporting guidelines, for instance the black triangle concept, which was already in place from the 1970s in the UK but introduced gradually in other European countries after 2013 [37]. Through the earlier introduction in the UK, HCPs might be more familiar with the concept of 'black triangle', which could explain a larger percentage of HCPs from the UK being motivated to report ADRs for medicines with a black triangle.

A motive to report that was important for HCPs in each of the countries was the reporting of severe and/or serious ADRs. Overall, $86 \%$ of the HCPs indicated this as a motive to report. This finding is similar to the previous study among nine European countries, where more than $80 \%$ of the HCPs 


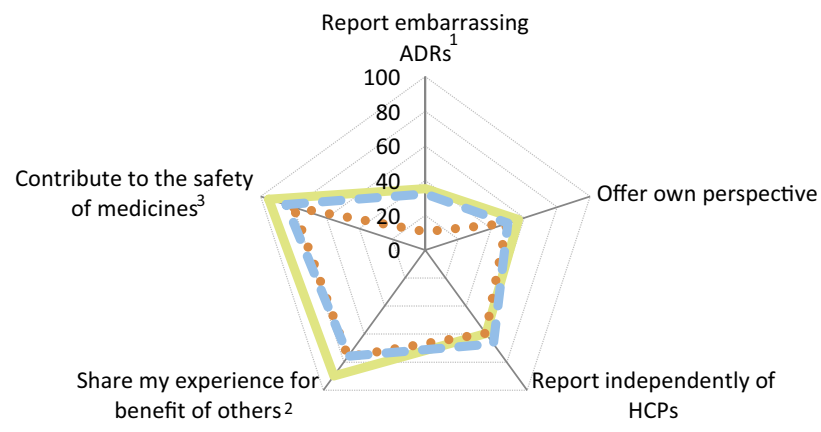

(A)

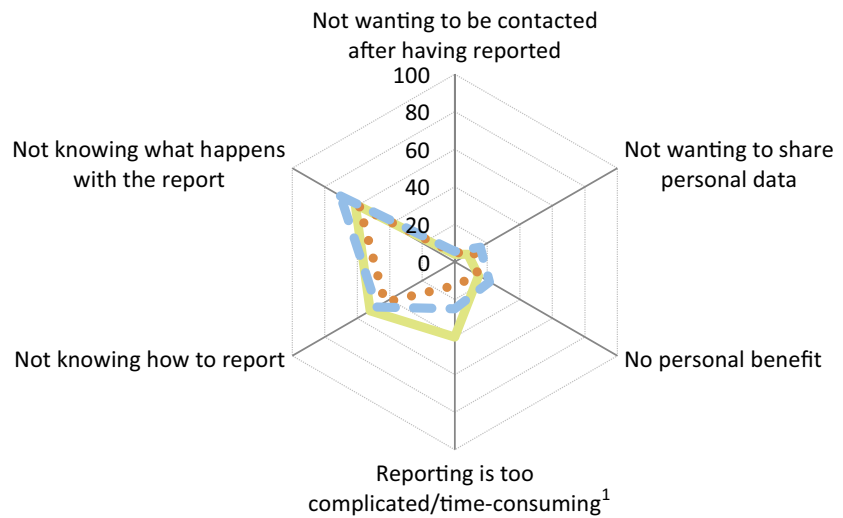

(B)
Fig. 2 Patients' responses to the question a 'In your opinion, what are the benefits of reporting side effects directly to the national agency?' (1 missing; 7 'none'; 21 other answers), and b 'What are, in your opinion, the drawbacks of reporting side effects directly to the national agency?' (112 'none'; 19 other answers). HCPs healthcare professionals, ADRs adverse drug reactions. Fig. 1A: ${ }^{1}$ Overall $\mathrm{P}<0.001$. $\mathrm{V}=0.27$. The Netherlands significantly different from
Croatia and the UK (both $\mathrm{P}<0.001$ ). ${ }^{2}$ Overall $\mathrm{P}=0.011 . \mathrm{V}=0.15$. The UK significantly different from Croatia $(P=0.006)$ and the Netherlands $(\mathrm{P}=0.005)$. ${ }^{3}$ Overall $\mathrm{P}=0.004$. $\mathrm{V}=0.16$. The UK significantly different from Croatia $(\mathrm{P}=0.012)$ and the Netherlands $(\mathrm{P}=0.001)$. Fig. 1B: ${ }^{1}$ Overall $\mathrm{P}<0.001$. $\mathrm{V}=0.25$. The Netherlands significantly different from Croatia $(\mathrm{P}=0.013)$ and the UK $(\mathrm{P}<$ $0.001)$

possibility of being able to report ADRs at all, and about which type of ADRs to report [40]. Awareness campaigns such as the European-wide campaign in November 2016 [31] can be used to increase the knowledge about such aspects. An important motive not to report an ADR for HCPs from all countries in this study was lack of time. Employing different strategies to facilitate reporting or reduce the time needed to complete the report should be considered to promote and increase ADR reporting. Time might be less of a problem for patients since the number of patients who indicated that reporting is too complicated or time-consuming was lower, particularly among patients from The Netherlands. A previous qualitative study [40] suggested asking patients more questions in the ADR report form than those asked to HCPs.

Our study also provides some country-specific suggestions to promote and increase ADR reporting. For stimulating ADR reporting among HCPs, the addition of the black triangle to specific medicines is a good strategy in the UK. In Croatia and The Netherlands, more awareness on the meaning of the black triangle symbol may be needed. For stimulating ADR reporting among patients, it is important to make clear that a report is not linked to the medical notes. This may be particularly helpful in Croatia and The Netherlands, since patients from these countries indicated that they would report if the report was not linked to their medical notes to which HCPs have access. In The Netherlands, an awareness campaign might be useful to stimulate the reporting of ADRs of medicines that are purchased via the internet instead of via the pharmacy, since fewer patients from The Netherlands than from Croatia and the UK would report 
ADRs for these medicines. Finally, the finding that more patients from Croatia and the UK than from The Netherlands saw it as beneficial to report embarrassing ADRs directly to the national agency could imply that, particularly in these countries, there should be more attention towards improving ADR communication between patients and HCPs. ADR reporting to national agencies will not have a direct effect on the treatment of the reporter. To improve the management of ADRs in clinical practice, it is important that patients and HCPs communicate about this matter.

\subsection{Strengths and Limitations}

A strength of this study is the assessment of motives among both HCPs and patients within the same study, and comparison of the results across countries. However, a limitation of the study is that baseline characteristics of the included population differed across the countries. A previous study showed differences among professions in reasons not to report an ADR [7]. Therefore, observed differences across the countries in our study may have been influenced by differences in the included population. On the other hand, only small differences in a previous study have been observed in the evaluation of motives between males and females and between those who are a member of a patient organization and those who are not [25]. Another limitation concerns the representativeness of the included HCPs and patients. In general, involvement in the topic of a survey can affect the attitude towards the survey, which may influence someone's intention to participate in the survey [41]. In our study, about $80 \%$ of the included patients had experienced an ADR and about half were aware of the possibility to report ADRs. These percentages are much higher than in a survey study conducted in Australia [42]. Furthermore, about $80 \%$ of the HCPs in our study had ever reported an ADR to the national pharmacovigilance center. It should be noted that the national agencies of the three countries assessed in this study were involved in the survey distribution. Although other distribution methods were also used, this involvement could imply that, in our study, there was an overrepresentation of people who were familiar with the national agency and/or had reported an ADR to the national agency. Finally, we were not able to calculate response rates due to the method of survey distribution.

\section{Conclusion}

This study shows that HCPs and patients from Croatia, The Netherlands, and the UK mostly have similar motives to report or not to report ADRs to the national agency. Focusing on these motives is a useful strategy to promote and increase ADR reporting across countries. However, relevant differences across the countries were observed in some motives. For HCPs, these concerned motives about legal obligation, black triangle medicines, and the reporting of well-known ADRs. For patients, these were a linkage between the report and the medical notes, complexity and time taken to report, medicines purchased on the internet, and the reporting of embarrassing ADRs. The knowledge about these differences can be used to fine-tune activities to promote and increase ADR reporting at a national level.

Supplementary Information The online version contains supplementary material available at https://doi.org/10.1007/s40264-021-01098-4.

\section{Declarations}

Funding This work was a collaboration between the Web-RADR project and the SCOPE Joint Action. The Web-RADR project has received support from the IMI Joint Undertaking (www.imi.europa.eu) under Grant Agreement no. 115632, the resources of which are composed of a financial contribution from the European Union's Seventh Framework Programme FP7/2007-2013 and European Federation of Pharmaceutical Industries and Associations (EFPIA) companies' in kind contribution, UK (www.imi.europa.eu). The SCOPE Joint Action was sponsored by the European Commission. Any opinions, conclusions, and proposals in the text are those of the authors and do not necessarily represent the views of the European Commission.

Conflicts of Interest Sieta T. de Vries, Petra Denig, Adriana Andrić, Marina Dimov Di Giusti, Alicia Ptaszynska-Neophytou, Linda Härmark, and Peter G.M. Mol have no conflicts of interest that are directly relevant to the contents of this study.

Availability of Data and Material/Code Availability The data and syntaxes used for this study are available from the corresponding author on reasonable request.

Author Contributions All authors contributed to the development and formulation of the research question. STdV, AA, MDDG, LH, and PGMM were involved in the distribution of the survey, and STdV and PGMM conducted the analyses. All authors contributed to the interpretation of the data. STdV, PD, and PGMM wrote the manuscript, and AA, MDDG, APN, and LH reviewed and edited the manuscript. All authors have read and approved the final version of the manuscript. This study was a collaboration between the SCOPE Joint Action and the Web-RADR project. The study objective was formulated by members of the SCOPE Work Package 4, and the survey questions were formulated in collaboration with members of the Web-RADR Work Package $3 \mathrm{~b}$ who performed the survey study. SCOPE Work Package 4 focused on national schemes for the spontaneous reporting of ADRs.

Ethical Approval This study was part of a wider project among HCPs and patients in Europe. The study protocol for the project was submitted to (a member of) an Ethics Committee in the UK, The Netherlands, and Germany. In the UK, approval was obtained from the UCL Research Ethics Committee (project ID number 6855/001). In The Netherlands, the Medical Ethics Committee of the University Medical Center Groningen (METcUMCG) determined that ethical approval was not needed for this study (reference number M16.191043), and in Germany, a review by an Ethics Committee was not needed; therefore, 
no further approval was deemed necessary for the other countries, including Croatia.

Consent to Participate Participants gave their implied consent to participate in the study by voluntarily completing the survey.

Consent to Publish Not applicable.

Disclaimer The views expressed in this article are the personal views of the authors and may not be understood or quoted as being made on behalf of, or reflecting the position of, the Medicines Evaluation Board or one of its committees.

Acknowledgments The authors would like to thank the HCPs and patients who responded to the survey. Acknowledgments go to the other Web-RADR Work Package $3 b$ and SCOPE Joint Action Work Package 4 members.

Open Access This article is licensed under a Creative Commons Attribution-NonCommercial 4.0 International License, which permits any non-commercial use, sharing, adaptation, distribution and reproduction in any medium or format, as long as you give appropriate credit to the original author(s) and the source, provide a link to the Creative Commons licence, and indicate if changes were made. The images or other third party material in this article are included in the article's Creative Commons licence, unless indicated otherwise in a credit line to the material. If material is not included in the article's Creative Commons licence and your intended use is not permitted by statutory regulation or exceeds the permitted use, you will need to obtain permission directly from the copyright holder. To view a copy of this licence, visit http://creativecommons.org/licenses/by-nc/4.0/.

\section{References}

1. Segal ES, Valette C, Oster L, Bouley L, Edfjall C, Herrmann P, et al. Risk management strategies in the postmarketing period: safety experience with the US and European bosentan surveillance programmes. Drug Saf. 2005;28(11):971-80. https://doi.org/10. 2165/00002018-200528110-00001.

2. Matos C, Harmark L, van Hunsel F. Patient reporting of adverse drug reactions: an international survey of national competent authorities' views and needs. Drug Saf. 2016;39(11):1105-16. https://doi.org/10.1007/s40264-016-0453-6.

3. Hazell L, Shakir SA. Under-reporting of adverse drug reactions: a systematic review. Drug Saf. 2006;29(5):385-96. https://doi.org/ 10.2165/00002018-200629050-00003.

4. Aagaard L, Strandell J, Melskens L, Petersen PS, Holme HE. Global patterns of adverse drug reactions over a decade: analyses of spontaneous reports to VigiBase. Drug Saf. 2012;35(12):117182. https://doi.org/10.1007/BF03262002.

5. Banovac M, Candore G, Slattery J, Houyez F, Haerry D, Genov $\mathrm{G}$, et al. Patient reporting in the EU: analysis of Eudra vigilance data. Drug Saf. 2017;40(7):629-45. https://doi.org/10.1007/ s40264-017-0534-1.

6. Margraff F, Bertram D. Adverse drug reaction reporting by patients: an overview of fifty countries. Drug Saf. 2014;37(6):409-19. https://doi.org/10.1007/s40264-014-0162-y.

7. Eland IA, Belton KJ, van Grootheest AC, Meiners AP, Rawlins $\mathrm{MD}$, Stricker BH. Attitudinal survey of voluntary reporting of adverse drug reactions. Br J Clin Pharmacol. 1999;48(4):623-7. https://doi.org/10.1046/j.1365-2125.1999.00060.x.

8. Oshikoya KA, Awobusuyi JO. Perceptions of doctors to adverse drug reaction reporting in a teaching hospital in Lagos,
Nigeria. BMC Clin Pharmacol. 2009;9:14. https://doi.org/10. 1186/1472-6904-9-14.

9. Santosh KC, Tragulpiankit P, Gorsanan S, Edwards IR. Attitudes among healthcare professionals to the reporting of adverse drug reactions in Nepal. BMC Pharmacol Toxicol. 2013;14:16. https:// doi.org/10.1186/2050-6511-14-16.

10. Cosentino M, Leoni O, Banfi F, Lecchini S, Frigo G. Attitudes to adverse drug reaction reporting by medical practitioners in a Northern Italian district. Pharmacol Res. 1997;35(2):85-8. https:// doi.org/10.1006/phrs.1996.0138.

11. Belton KJ, Lewis SC, Payne S, Rawlins MD, Wood SM. Attitudinal survey of adverse drug reaction reporting by medical practitioners in the United Kingdom. Br J Clin Pharmacol. 1995;39(3):223-6. https://doi.org/10.1111/j.1365-2125.1995. tb04440.x.

12. Green CF, Mottram DR, Rowe PH, Pirmohamed M. Attitudes and knowledge of hospital pharmacists to adverse drug reaction reporting. Br J Clin Pharmacol. 2001;51(1):81-6. https://doi.org/ 10.1046/j.1365-2125.2001.01306.x.

13. Mirbaha F, Shalviri G, Yazdizadeh B, Gholami K, Majdzadeh R. Perceived barriers to reporting adverse drug events in hospitals: a qualitative study using theoretical domains framework approach. Implement Sci. 2015;10:110. https://doi.org/10.1186/ s13012-015-0302-5.

14. Milstien JB, Faich GA, Hsu JP, Knapp DE, Baum C, Dreis MW. Factors affecting physician reporting of adverse drug reactions. Drug Inf J. 1986;29:157-64. https://doi.org/10.1177/0092861586 02000207.

15. John LJ, Arifulla M, Cheriathu J, Sreedharan J. Reporting of adverse drug reactions: a study among clinicians. J Appl Pharm Sci. 2012. https://doi.org/10.1186/2008-2231-20-44.

16. Gurmesa LT, Dedefo MG. Factors affecting adverse drug reaction reporting of healthcare professionals and their knowledge, attitude, and practice towards ADR reporting in Nekemte Town, West Ethiopia. Biomed Res Int. 2016. https://doi.org/10.1155/ 2016/5728462.

17. De Angelis A, Colaceci S, Giusti A, Vellone E, Alvaro R. Factors that condition the spontaneous reporting of adverse drug reactions among nurses: an integrative review. J Nurs Manag. 2016;24(2):151-63. https://doi.org/10.1111/jonm.12310.

18. Lopez-Gonzalez E, Herdeiro MT, Figueiras A. Determinants of under-reporting of adverse drug reactions: a systematic review. Drug Saf. 2009;32(1):19-31. https://doi.org/10.2165/00002018200932010-00002.

19. Varallo FR, De Guimarães SOP, Abjaude SAR, De Mastroianna PC. Causes for the underreporting of adverse drug events by health professionals: a systematic review. Rev Esc Enferm USP. 2014;48(4):739-47. https://doi.org/10.1590/s0080-6234201400 00400023.

20. Abjaude SAR, Mieli SF, Magalhães ZR, Pereira LRL. Factors that motivate healthcare professionals to report adverse drug events: a systematic review. Pharm Med. 2017;31(1):13-20. https://doi. org/10.1007/s40290-016-0174-2.

21. de Langen J, van Hunsel F, Passier A, de van den Berg LJ, van Grootheest K. Adverse drug reaction reporting by patients in the Netherlands: three years of experience. Drug Saf. 2008;31(6):51524. https://doi.org/10.2165/00002018-200831060-00006.

22. Inacio $\mathrm{P}$, Cavaco $\mathrm{A}$, Airaksinen $\mathrm{M}$. The value of patient reporting to the pharmacovigilance system: a systematic review. $\mathrm{Br} \mathrm{J}$ Clin Pharmacol. 2017;83(2):227-46. https://doi.org/10.1111/bcp. 13098.

23. Blenkinsopp A, Wilkie P, Wang M, Routledge PA. Patient reporting of suspected adverse drug reactions: a review of published literature and international experience. Br J Clin Pharmacol. 2007;63(2):148-56. https://doi.org/10.1111/j.1365-2125.2006. 02746.x 
24. Al Dweik R, Stacey D, Kohen D, Yaya S. Factors affecting patient reporting of adverse drug reactions: a systematic review. Br J Clin Pharmacol. 2017;83(4):875-83. https://doi.org/10.1111/bcp. 13159.

25. van Hunsel F, van der Welle C, Passier A, van Puijenbroek E, van Grootheest $\mathrm{K}$. Motives for reporting adverse drug reactions by patient-reporters in the Netherlands. Eur J Clin Pharmacol. 2010;66(11):1143-50. https://doi.org/10.1007/ s00228-010-0865-7.

26. Jarernsiripornkul N, Patsuree A, Krska J. Public confidence in ADR identification and their views on ADRreporting: mixed methods study. Eur J Clin Pharmacol. 2017;73(2):223-31. https:// doi.org/10.1007/s00228-016-2155-5.

27. Matos C, van Hunsel F, Joaquim J. Are consumers ready to take part in the pharmacovigilance system? A Portuguese preliminary study concerning ADR reporting. Eur J Clin Pharmacol. 2015;71(7):883-90. https://doi.org/10.1007/s00228-015-1867-2.

28. Pierce CE, de Vries ST, Bodin-Parssinen S, Harmark L, Tregunno P, Lewis DJ, et al. Recommendations on the use of mobile applications for the collection and communication of pharmaceutical product safety information: lessons from IMI WEBRADR. Drug Saf. 2019;42(4):477-89. https://doi.org/10.1007/ s40264-019-00813-6.

29. Ghosh R, Lewis D. Aims and approaches of Web-RADR: a consortium ensuring reliable ADR reporting via mobile devices and new insights from social media. Expert Opin Drug Saf. 2015;14(12):1845-53. https://doi.org/10.1517/14740338.2015. 1096342 .

30. de Vries ST, Denig P, Lasheras Ruiz C, Houyez F, Wong L, Sutcliffe A, et al. Interest in a mobile app for two-way risk communication: a survey study among European healthcare professionals and patients. Drug Saf. 2018;41(7):697-712. https://doi.org/10. 1007/s40264-018-0648-0.

31. Radecka A, Loughlin L, Foy M, de Ferraz MVG, Sarinic VM, Di Giusti MD, et al. Enhancing pharmacovigilance capabilities in the EU regulatory network: The SCOPE Joint Action. Drug Saf. 2018;41(12):1285-302. https://doi.org/10.1007/ s40264-018-0708-5.

32. Biriell C, Edwards IR. Reasons for reporting adverse drug reactions-some thoughts based on an international review. Pharmacoepidemiol Drug Saf. 1997;6(1):21-6. https://doi.org/10.1002/ (SICI) 1099-1557(199701)6:1\%3c21::AID-PDS259\%3e3.0. $\mathrm{CO} ; 2-\mathrm{I}$.

33. Kim HY. Statistical notes for clinical researchers: Chi-squared test and Fisher's exact test. Restor Dent Endod. 2017;42(2):152-5. https://doi.org/10.5395/rde.2017.42.2.152.
34. Directive 2001/83/EC of the European Parliament and of the Council of 6 November 2001 on the Community code relating to medicinal products for human use. https://ec.europa.eu/health/ sites/health/files/files/eudralex/vol-1/dir_2001_83_consol_2012/ dir_2001_83_cons_2012_en.pdf. Accessed 29 Sep 2020

35. Belton KJ. Attitude survey of adverse drug-reaction reporting by health care professionals across the European Union. The European Pharmacovigilance Research Group. Eur J Clin Pharmacol. 1997;52(6):423-7. https://doi.org/10.1007/s002280050314.

36. Oosterhuis I, Taavola H, Tregunno PM, Mas P, Gama S, Newbould V, et al. Characteristics, quality and contribution to signal detection of spontaneous reports of adverse drug reactions via the WEB-RADR mobile application: a descriptive cross-sectional study. Drug Saf. 2018;41(10):969-78. https://doi.org/10.1007/ s40264-018-0679-6.

37. SCOPE Work Package 4. ADR Collection. Identification, management and raising awareness of ADR reports for drugs subject to additional monitoring. https://www.ema.europa.eu/en/documents/ other/scope-training-identification-management-raising-aware ness-adr-reports-drugs-subject-additional_en.pdf. Accessed 29 Sep 2020

38. Wysowski DK, Nourjah P, Swartz L. Bleeding complications with warfarin use: a prevalent adverse effect resulting in regulatory action. Arch Intern Med. 2007;167(13):1414-9. https://doi.org/ 10.1001/archinte.167.13.1414.

39. HALMED Agency for Medicinal Products and Medical Devices of Croatia. Reporting of adverse drug reactions by patients - Croatian experience. https://www.ema.europa.eu/en/documents/prese ntation/presentation-reporting-adverse-drug-reactions-patientscroatian-experience-marina-giusti_en.pdf. Accessed 29 Sep 2020

40. de Vries ST, Wong L, Sutcliffe A, Houyez F, Ruiz CL, Mol PG, et al. Factors influencing the use of a mobile app for reporting adverse drug reactions and receiving safety information: a qualitative study. Drug Saf. 2017;40(5):443-55. https://doi.org/10.1007/ s40264-016-0494-x.

41. Fang J, Wen C. Predicting potential respondents' decision to participate in web surveys. Int J Serv Technol Manag. 2012;18:1632. https://doi.org/10.1504/IJSTM.2012.049013.

42. Robertson J, Newby DA. Low awareness of adverse drug reaction reporting systems: a consumer survey. Med J Aust. 2013;199(10):684-6. https://doi.org/10.5694/mja13.10069.

\section{Authors and Affiliations}

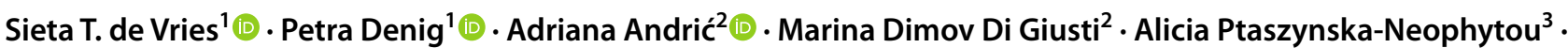 Linda Härmark ${ }^{4}{ }^{\infty}$. Peter G. M. Mol ${ }^{1,5}$ on behalf of the IMI Web-RADR Work Package $3 b$ Consortium and SCOPE Joint Action Work Package 4}

1 Department of Clinical Pharmacy and Pharmacology, University of Groningen, University Medical Center Groningen, Groningen, The Netherlands

2 Agency for Medicinal Products and Medical Devices of Croatia (HALMED), Zagreb, Croatia

3 Medicines and Healthcare Products Regulatory Agency, London, UK
4 Netherlands Pharmacovigilance Centre Lareb, 's-Hertogenbosch, The Netherlands

5 Dutch Medicines Evaluation Board, Utrecht, The Netherlands 